\title{
Minor to Moderate Side Effects of Pfizer-BioNTech COVID-I 9 Vaccine Among Saudi Residents: A Retrospective Cross-Sectional Study
}

\author{
Nagla A El-Shitany iD ${ }^{1,2}$ \\ Steve Harakeh iD ${ }^{3}$ \\ Shaimaa M Badr-Eldin ${ }^{4,5}$ \\ Amina M Bagher' \\ Basma Eid' \\ Haifa Almukadi ${ }^{\prime}$ \\ Badrah S Alghamdi ${ }^{6}$ \\ Ahlam A Alahmadi \\ Nibal A Hassan ${ }^{8}$ \\ Nariman Sindi ${ }^{9}$ \\ Samar A Alghamdi ${ }^{10}$ \\ Hailah M Almohaimeed " \\ Zuhair M Mohammedsaleh ${ }^{12}$ \\ Turki M Al-Shaikh ${ }^{13}$ \\ Mohammed S Almuhayawi ${ }^{14}$ \\ Soad S Ali ${ }^{15}$ \\ Manal El-Hamamsy ${ }^{16,17}$
}

'Department of Pharmacology and Toxicology, Faculty of Pharmacy, King Abdulaziz University, Jeddah, Saudi Arabia; ${ }^{2}$ Department of Pharmacology and Toxicology, Faculty of Pharmacy, Tanta University, Tanta, Egypt; ${ }^{3}$ Special Infectious Agents Unit, King Fahd Medical Research Center, Yousef Abdullatif Jameel Chair of Prophetic Medicine Application, Faculty of Medicine, King Abdulaziz University, Jeddah, Saudi Arabia; ${ }^{4}$ Department of Pharmaceutics, Faculty of Pharmacy, King Abdulaziz University, Jeddah, Saudi Arabia; ${ }^{5}$ Department of Pharmaceutics and Industrial Pharmacy, Faculty of Pharmacy, Cairo University, Cairo, Egypt;

${ }^{6}$ Department of Physiology, Neuroscience Unit. Faculty of Medicine, King Abdulaziz University, Jeddah, Saudi Arabia; ${ }^{7}$ Department of Biological Sciences, Faculty of Science, King Abdulaziz University, Jeddah, Saudi Arabia; ${ }^{8}$ Department of Biology, Faculty of Science, Taif University, Taif, Saudi Arabia 'Department of Medical Laboratory Technology, Faculty of Applied Medical Sciences, King Abdulaziz University, Jeddah, Saudi Arabia; ${ }^{10}$ Department of Oral Biology, Faculty of Dentistry, King Abdulaziz University, Jeddah, Saudi Arabia; "Department of Basic Science, Faculty of Medicine, Princess Nourah Bint Abdulrahman University, Riyadh, Saudi Arabia; ${ }^{2}$ Department of Medical Laboratory Technology, Faculty of Applied Medical Sciences, University of Tabuk, Tabuk, Saudi Arabia; ${ }^{13}$ Department of Biology, College of Science and Arts at Khulis, University of Jeddah, Jeddah, Saudi Arabia; ${ }^{14}$ Department of Microbiology and Medical Parasitology, Faculty of Medicine, King Abdulaziz University, Jeddah, 21589 , Saudi Arabia; ${ }^{15}$ Department of Histology, Faculty of Medicine, Assiut University, Assiut Egypt; ${ }^{16}$ Department of Pharmacy Practice, Faculty of Pharmacy, King Abdulaziz University, Jeddah, Saudi Arabia; ${ }^{17}$ Department of Clinical Pharmacy, Faculty of Pharmacy, Ain Shams University, Cairo, Egypt

Correspondence: Nagla A El-Shitany Department of Pharmacology and Toxicology, Faculty of Pharmacy, King Abdulaziz University, Al-Sheekh Kamal Street, Alsulimaniah, Jeddah, Saudi Arabia Tel +966545923440

Email nagla fouad@yahoo.com
Background: The Pfizer-BioNTech COVID-19 vaccine has recently received emergency approval from the US FDA. The mRNA technology was used to manufacture the Pfizer vaccine; however, as a pioneering technology that has never been used in the manufacture of vaccines, many people have concerns about the vaccine's side effects. Thus, the current study aimed to track the short-term side effects of the vaccine.

Methods: The information in this study was gathered by a Google Form-questionnaire (online survey). The results included the responses of 455 individuals, all of whom are Saudi Arabia inhabitants. Adverse effects of the vaccine were reported after the first and the second doses.

Results: The most common symptoms were injection site pain, headaches, flu-like symptoms, fever, and tiredness. Less common side effects were a fast heartbeat, whole body aches, difficulty breathing, joint pain, chills, and drowsiness. Rare side effects include Bell's palsy and lymph nodes swelling and tenderness. Flu-like symptoms were more common among those under 60 years of age, while injection site pain was more frequent among recipients who were 60 years and older. The study revealed a significant increase in the number of females who suffered from the vaccine side effects compared to males. Difficulty of breathing was more reported among recipients who had been previously infected with the coronavirus compared to those who had not been previously infected.

Conclusion: Most of the side effects reported in this study were consistent with Pfizer's fact sheet for recipients and caregivers. Further studies are required to determine the long-term side effects.

Keywords: Pfizer-BioNTech COVID-19 vaccine, side effects, injection site pain, hypersensitivity, flu-like symptoms, online survey

\section{Background}

Coronavirus 2 (SARS-CoV-2), a new member of the human coronaviruses family, was identified in 2019 as the causative agent of a new disease outbreak in China associated with severe medical complications and even death in some cases. ${ }^{1}$ In March 2020, the World Health Organization (WHO) announced the novel disease outbreak as a pandemic. ${ }^{2}$

The unavailability of a vaccine or other efficacious therapeutic option has required all the nations worldwide to combat the pandemic's spread. Several precautionary strategies, which include lockdown, social distancing, wearing facemasks, and travel limitations, have been applied to stop this pandemic. However, vaccine development is perhaps the best hope for stopping this pandemic. ${ }^{3}$ 
On December 11, 2020, the US Food and Drug Administration (FDA) authorized the emergency use of the Pfizer-BioNTech COVID-19 vaccine. The vaccination requires two shots given at least 21 days apart. It has been reported that the maximum efficacy, about $95 \%$, can be reached one week following the second dose. The vaccine could be utilized for people ages 16 years and older. ${ }^{4}$ Authorization for emergency utilization has also been given to the Moderna COVID-19 vaccine for the use in people ages 18 and older with an efficacy rate of $94.1 \%$. The Moderna vaccine also involves two shots that are administered 28 days apart. ${ }^{5}$

The technology for both the Pfizer-BioNTech and Moderna COVID-19 vaccines relies on messenger RNA (mRNA). The coronavirus possesses a spike-shaped surface structure called an S protein, and the COVID-19 mRNA vaccines give instructions on the way to produce a harmless piece of an S protein. Following vaccination, cells start making more of the protein pieces and introducing them to the coronavirus's surfaces. The immune system will perceive that the protein does not belong there and start to create an immune response and form antibodies. ${ }^{6}$

Sometimes after a vaccination, the process of building immunity can cause adverse symptoms. The PfizerBioNTech COVID-19 vaccine can induce mild side effects following the first and/or the second shot, including pain, redness or swelling at the injection site, tiredness, headaches, chills, muscle and joint aches, and fever. These symptoms could be an indication that the body is developing the desired immunity for protection. ${ }^{7}$

The success of the vaccination strategies relies partly on population's conceptions of the vaccines' benefits and risks and on their related trust in the vaccination. Researchers believe that refusing or delaying of the vaccination is a result of a lack of knowledge about the relative benefit-to-risk ratio of the vaccination, ${ }^{8-10}$ which is a status that was defined as vaccine hesitancy by the WHO Strategic Advisory Group of Experts on Immunization in 2015. ${ }^{11}$ WHO recognized this hesitancy as one of the major global health threats in $2019 .{ }^{12}$

Pfizer-BioNTech's COVID-19 vaccine is an actual fight against the pandemic. However, since the mRNA technique is new in vaccine manufacturing, one cannot predict its consequences. Hence, studies are essential to follow the vaccine's side effects. Besides, understanding the Pfizer-BioNTech COVID-19 vaccine's risk and declaring it to the public are required to raise the acceptance of the vaccination.
This study aimed to evaluate the short-term side effects after receiving the first, the second, and/or both doses of the Pfizer-BioNTech COVID-19 vaccine in a sample of 16 years and older residents in Saudi Arabia. The study intended to identify which age group is most vulnerable to the short-term side effects.

\section{Methods}

\section{Study Design}

A cross-sectional (online survey) study was conducted in a retrospective manner. The subjects participating in the study were residents in Saudi Arabia. Saudi Arabia began its campaign to vaccinate the population against the emerging coronavirus on December 17, 2020, with the PfizerBioNTech vaccine. The present study was conducted over the period between January 10 and 21, 2021. A questionnaire, designed on Google Forms, was written in the Arabic language (the scientific terms for the symptoms were written and explained in the public language) and delivered to participants via social media (primarily Facebook and WhatsApp). Communication between the investigators and the subjects participating in the study was established via electronic mail when required. The participants were not given any incentives to participate. Still, the importance of the research in educating the community about the vaccine's side effects was clarified. Their participation may also prompt everyone else to receive the vaccine after comparing the acceptable side effects with the disease's severity. The settings were adjusted to that each participant sent only one response. Still, sometimes some participants asked to be allowed to send more than one response, as they were also filling out a questionnaire about their elderly relatives who could not handle the electronic questionnaires. Responses were carefully reviewed to ensure that responses were not repeated.

The questionnaire comprised two categories of inquiries. The first one covers background data of the subject, such as nationality, gender, age, educational level, and previous infection with SARS-CoV-2. The second group of inquiries focused on COVID-19 vaccine-related data. The survey asks what type of COVID-19 vaccine the participant received and whether the participant received one or two doses of the vaccine. This section also asks what side effects are associated with administering the COVID-19 vaccine, in addition to the timing of the appearance of these effects (after the first or second dose). Regarding these side effects, the participants 
were asked to choose from symptoms including temporary face paralysis (Bell's palsy), flu-like symptoms, pain at the site of injection, dyspnea, headache, and tachycardia and to state any other symptoms that they experienced following the vaccination. All participants were permitted to terminate the survey at any time of the study. All precautionary actions were applied to preserve the data's confidentiality. The questionnaire was pre-tested for validity. First two experts assessed all the questions individually and minor modifications were made based on their based on their feedback. Then the questionnaire was given to three faculty members at King Abdulaziz University, and they were asked to rate each of the questions by using a 5-point Likert scale ranging from 1 to 4 $(1=$ very important, $2=$ important, $3=$ moderately important, and $4=$ not important). The validity was then evaluated by calculating the Item-Content Validity Index (I-CVI). The results showed excellent content validity $(\mathrm{I}-\mathrm{CVI}=1)$.

\section{Inclusion Criteria}

Inclusion criteria included the administration of at least one dose of the Pfizer-BioNTech COVID-19 vaccine.

\section{Exclusion Criteria}

Participants who were not vaccinated against COVID-19 or who had received a vaccine other than one made by the Pfizer Company were excluded.

\section{Sample Size}

The minimum sample size for conducting this survey was 384 with a $5 \%$ margin of error and a $95 \%$ confidence level. We have hypothesized that the prevalence of the side effects in the population is $50 \% \pm 5$ (the reported side effects by FDA ranged from $14.2 \%$ to $84.1 \%$ ). In this study, 467 subjects completed the COVID-19 vaccine questionnaire. The number of participants that who satisfied the inclusion and exclusion criteria was 455 (Figure 1).

\section{Study Variables}

The age of the participants and the timing of the appearance of COVID-19 vaccine side effects were explored as independent variables. The various adverse reactions observed as side effects following a vaccination were considered as the dependent variable.

\section{Statistical Analysis}

Descriptive statistics were performed for the collected data. The responses were displayed as frequency (counts and/or percentage). A chi-squared test was employed for statistical analysis. The data were statistically analyzed using Prism ${ }^{\circledR}$ (version 8.4.0, GraphPad Software Inc., La Jolla, CA, USA). The level of significance was set at $\mathrm{p} \leq 0.05$.

\section{Results}

\section{The Study Participants' Demographic Characteristics, COVID-19 Past Infection, Type of Vaccine, Number of Vaccine Doses, and the Onset of Side Effects}

For this study, 455 individuals, all of them of Arab descent, participated. Saudi citizens were the majority of the participants $(425,93.4 \%)$, and the minority came from other nationalities $(30,6.6 \%)$. Women were the majority of the participants $(292,64.2 \%)$, while men constituted the minority of participants $(163,35.8 \%)$. It was found that $299(65.7 \%)$ participants were younger than 60 years of age, and $156(34.3 \%)$ were 60 years or older. Most of the study population was made up of people with the university $(207,45.5 \%)$ and postuniversity $(183,40.2 \%)$ education levels, while the minority were those with pre-university $(65,14.3 \%)$ education. The study population's past medical history revealed that, while a minority of them $(19,4.2 \%)$ had previously been infected with the SARS-CoV-2 virus, most participants $(436,95.8 \%)$ had never been infected. All study participants $(455,100 \%)$ had received their first PfizerBioNTech COVID-19 vaccine dose, while 252 (55.4\%) had received both doses. Of the participants, 237 (52\%) individuals reported the presence or absence of the side effects after receiving their first dose of the vaccine, 124 (27.3\%) after receiving their second dose, and 94 (20.7\%) people after receiving both doses, Table 1.

\section{Reported COVID-19 Vaccine Side Effects and Their Correlation with the First and Second Doses}

This study results showed a significant increase $(\mathrm{p}<$ 0.001 ) in the number of subjects who were suffering side effects after receiving the second dose of the vaccine (122, 98.4\%) compared to those who reported side effects after 
the first dose $(202,85.2 \%)$ or after both doses $(68,72.3 \%)$, Table 2 and Figure 2.

The results also showed a significant increase $(\mathrm{p}<$ 0.05 ) in the number of participants who reported injection site side effects after receiving the second dose of the vaccine $(100,80.6 \%)$ compared to the number of participants who reported local side effects of the vaccine after receiving the first dose $(167,70.5 \%)$ or both doses $(60$, $63.8 \%)$. After the first vaccine dose, $4(1.7 \%)$ participants reported arm and shoulder pain, and $2(0.8 \%)$ participants reported swelling and redness at the injection site. However, no one reported these symptoms after the second or both doses of the vaccine. There was a significant increase $(p<0.05)$ in the number of participants who experienced injection site pain after receiving the second dose of the vaccine $(100,80.6 \%)$ compared to those who received their first dose $(161,67.9 \%)$ or who experienced this symptom after both doses $(60,63.8 \%)$, Table 2 and Figure 3.

These results showed a significant increase $(\mathrm{p}<0.01)$ in the number of individuals who reported hypersensitivity symptoms after receiving the second dose of the vaccine $(18,14.5 \%)$ compared to the number of individuals who reported hypersensitivity symptoms after receiving the first dose $(19,8.0 \%)$ or both doses $(3,3.2 \%)$. There was a significant increase $(p<0.05)$ in the number of individuals who reported a fast heartbeat after receiving the second dose of the vaccine $(11,8.9 \%)$ compared to the number of individuals who reported a fast heartbeat after receiving the first dose $(7,3.0 \%)$ or both doses $(3$, $3.2 \%)$. Nine (3.8\%) individuals reported shortness of breath after the first dose of the vaccine, and $5(4.0 \%)$ individuals after the second dose. One individual $(0.8 \%)$ reported a bad rash all over the body after the second vaccine dose, while one other individual $(0.4 \%)$ also reported a severe body allergy after the first vaccine dose. A burning sensation in the eye was reported by 2 $(0.8 \%)$ individuals after the first dose of the vaccine while only one individual $(0.8 \%)$ after the second dose of the vaccine, Table 2 and Figure 3.

The results showed a significant increase $(p<0.001)$ in the number of persons who reported bone and muscle pain after receiving the second dose of the vaccine $(22,17.7 \%)$ compared to the number of persons who reported bone and muscle pain after receiving the first dose $(9,3.8 \%)$ or both doses $(7,7.4 \%)$. There was a significant increase $(\mathrm{p}<$ 0.01 ) in the number of persons who reported whole body pain and joint pain after receiving the second dose of the

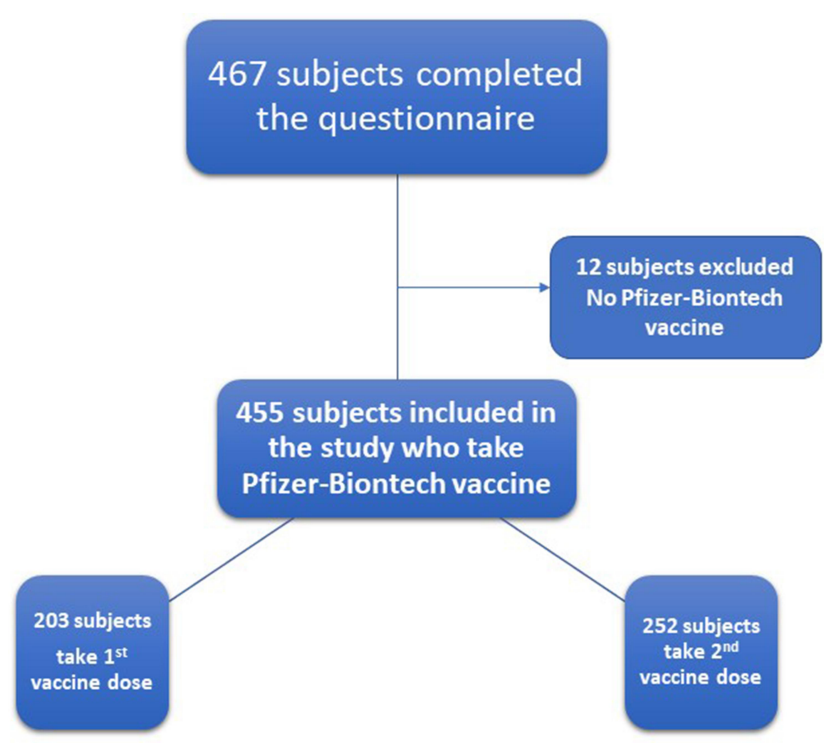

Figure I Flowchart for the COVID-19 vaccine questionnaire participants.

vaccine, $8(6.5 \%)$ and $5(4.0 \%)$ compared to the number of persons who reported whole body pain and joint pain after receiving the first dose, $2(0.8 \%)$ and $0(0.0 \%$ or both doses, $5(5.3 \%)$ and 1 (1.1\%). After the first vaccine dose, $2(0.8 \%)$ persons reported muscle pain, $3(1.3 \%)$ reported bone pain, and $2(0.8 \%)$ reported back pain. After the second vaccine dose, $1(0.8 \%)$ person reported muscle pain, $1(0.8 \%)$ reported muscle relaxation, 2 (1.6\%) reported bone pain and $3(2.4 \%)$ reported back pain. One person $(1.1 \%)$ reported muscle pain after his first and second vaccine doses, Table 2 and Figure 3.

The results showed a significant increase $(p<0.001)$ in the number of individuals who reported total flu-like symptoms after receiving the second dose of the vaccine $(112,90.3 \%)$ compared to the number of individuals who reported total flu-like symptoms after receiving the first dose $(77,32.5 \%)$ or both doses $(29,30.9 \%)$. There was a significant increase $(p<0.001)$ in the number of individuals who reported flu symptoms after receiving the second dose of the vaccine $(36,29 \%)$ compared to the number of individuals who reported flu symptoms after receiving the first dose $(26,11.0 \%)$ or both doses $(7$, $7.4 \%)$. Besides, there was a significant increase $(\mathrm{p}<$ 0.001 ) in the number of individuals who reported fever after receiving the second dose of the vaccine $(23,18.5 \%)$ compared to the number of individuals who reported fever after receiving the first dose $(3,1.3 \%)$ or both doses (4, $4.3 \%)$. After the first vaccine dose, $2(0.8 \%)$ individuals reported chills, $2(0.8 \%)$ reported sore throat and $1(0.4 \%)$ 
Table I The Study Participants' Demographic Characteristics, COVID-19 Past Infection, Type of Vaccine, Number of Vaccine Doses, and the Onset of Side Effects

\begin{tabular}{|c|c|}
\hline \multirow[t]{2}{*}{ Characteristics } & Frequency ( $n$ and \%) \\
\hline & All Participants $(n=455)$ \\
\hline \multicolumn{2}{|l|}{ Nationality } \\
\hline Saudi & $425(93.4 \%)$ \\
\hline Non-Saudi & $30(6.6 \%)$ \\
\hline \multicolumn{2}{|l|}{ Sex } \\
\hline Male & $163(35.8 \%)$ \\
\hline Female & $292(64.2 \%)$ \\
\hline \multicolumn{2}{|l|}{ Age (year) } \\
\hline$<60$ & $299(65.7 \%)$ \\
\hline$\geq 60$ & $156(34.3 \%)$ \\
\hline \multicolumn{2}{|l|}{ Education Level } \\
\hline Pre-university education & $65(14.3 \%)$ \\
\hline University education & 207 (45.5\%) \\
\hline Post-university education & $183(40.2 \%)$ \\
\hline \multicolumn{2}{|l|}{ Previous Infection with SARS-CoV-2 } \\
\hline Infected & $19(4.2 \%)$ \\
\hline Not infected & $436(95.8 \%)$ \\
\hline \multicolumn{2}{|l|}{ Type of COVID-19 Vaccine } \\
\hline Pfizer-BioNTech vaccine & $455(100 \%)$ \\
\hline \multicolumn{2}{|l|}{ Inoculated Vaccine Dose } \\
\hline Ist dose & $203(44.6 \%)$ \\
\hline Ist and 2 nd dose & $252(55.4 \%)$ \\
\hline \multicolumn{2}{|l|}{ Onset of Symptoms } \\
\hline After Ist vaccine dose & $237(52.0 \%)$ \\
\hline After 2nd Vaccine dose & $124(27.3 \%)$ \\
\hline After both Ist and 2 nd dose & 94 (20.7\%) \\
\hline
\end{tabular}

Note: Results were offered as frequency (number $(n)$ and percentage $(\%)$ ).

reported chest infection. After the second vaccine dose, 5 $(4.0 \%)$ individuals reported chills. One individual (1.1\%) reported chills after his first and second vaccine doses, Table 2 and Figure 3.

The results also showed a significant increase $(\mathrm{p}<0.001)$ in the number of participants who reported fatigue after receiving the second dose of the vaccine $(25$, $20.1 \%$ ) compared to the number of participants who reported fatigue after receiving the first dose $(20,8.4 \%)$ or both doses $(3,3.2 \%)$. Besides, there was a significant increase $(\mathrm{p}<0.05)$ in the number of participants who reported a desire to sleep after receiving the second dose of the vaccine $(4,3.2 \%)$ compared to the number of individuals who reported a desire to sleep after receiving the first dose $(1,0.4 \%)$ or both doses $(0,0 \%)$. After the first vaccine dose, 15 (6.3\%) participants reported tiredness, and 4 (1.7\%) reported dizziness and giddiness. After the second vaccine dose, 19 (15.3\%) participants reported tiredness, and $2(1.6 \%)$ reported dizziness and giddiness. Three participants (3.2\%) reported tiredness after their first and second vaccine doses, Table 2 and Figure 3.

The results also showed a significant increase $(p<0.05)$ in the number of persons who reported GIT symptoms after receiving the first dose of the vaccine (14, $5.9 \%)$ compared to the number of persons who reported GIT symptoms after receiving the second dose $(2,1.6 \%)$ or both doses $(1,1.1 \%)$. After the first vaccine dose, 4 (1.7\%) persons reported nausea and vomiting, $2(0.8 \%)$ reported gastroesophagitis, 7 (3.0\%) reported diarrhea, and $1(0.4 \%)$ reported a loss of appetite. After the second vaccine dose, $1(0.8 \%)$ reported nausea and vomiting, and $1(0.8 \%)$ reported diarrhea. One person (1.1\%) reported nausea and vomiting after his first and second vaccine doses, Table 2 and Figure 3.

After the first vaccine dose, $3(1.3 \%)$ individuals reported Bell's palsy, and $1(0.4 \%)$ reported body tingling, Table 2 and Figure 3.

The results also showed a significant increase $(\mathrm{p}<0.05)$ in the number of persons who reported miscellaneous symptoms after receiving the second dose of the vaccine $(4,3.2 \%)$ compared to the number of persons who reported miscellaneous symptoms after receiving the first dose $(1,0.4 \%)$ or both doses $(0,0 \%)$. Only one $(0.4 \%)$ person reported a burning sensation with urination after his first vaccine dose. After the second vaccine dose, 2 (1.6\%) persons reported lymph nodes tenderness and swelling, 1 $(0.8 \%)$ person reported elevated blood pressure, and 1 $(0.8 \%)$ reported blurred vision, Table 2 and Figure 3.

\section{Reported COVID-19 Vaccine Side Effects and Their Correlation with Participants' Ages}

Table 3 and Figure 4 showed the reported COVID-19 vaccine side effects distribution according to the participants' ages. The study results showed no significant difference between those who were under the age of 60 years and suffering from COVID-19 vaccine side effects and those over the age of 60 . On the other hand, there was a significant increase $(p<0.01)$ in the number of people who reported local symptoms and injection site pain over 60 years of age, $128(82.0 \%)$ and $126(80.8 \%)$ compared to those under 60 years old, 209, (69.9\%) and 205 
Table 2 The Reported COVID-I 9 Vaccine Side Effects and Their Correlation with the Vaccine's First, Second, and Both Doses

\begin{tabular}{|c|c|c|c|c|}
\hline & \multicolumn{4}{|l|}{ Frequency ( $n$ and \%) } \\
\hline & Ist Dose $(n=237)$ & 2nd Dose $(n=124)$ & Ist \& 2nd Doses $(n=94)$ & Chi-Square $p$ value \\
\hline \multicolumn{5}{|l|}{ Presence of Symptoms } \\
\hline Presence & $202(85.2 \%)$ & $122(98.4 \%)$ & $68(72.3 \%)$ & $<0.000 I^{* * * *}$ \\
\hline Absence & $35(14.8 \%)$ & $2(1.6 \%)$ & $26(27.7 \%)$ & \\
\hline Local Symptoms & $167(70.5 \%)$ & $100(80.6 \%)$ & $60(63.8 \%)$ & $0.0139 *$ \\
\hline Arm pain & $4(1.7 \%)$ & $0(0 \%)$ & $0(0 \%)$ & 0.1563 \\
\hline Injection site pain & $161(67.9 \%)$ & $100(80.6 \%)$ & $60(63.8 \%)$ & $0.0116 *$ \\
\hline Injection site swelling and redness & $2(0.8 \%)$ & $0(0 \%)$ & $0(0 \%)$ & 0.3907 \\
\hline Hypersensitivity Symptoms & $19(8.0 \%)$ & $18(14.5 \%)$ & $3(3.2 \%)$ & $0.0099 * *$ \\
\hline A fast heartbeat & 7 (3.0\%) & II (8.9\%) & $3(3.2 \%)$ & $0.0299 *$ \\
\hline Difficulty breathing & $9(3.8 \%)$ & $5(4.0 \%)$ & $0(0 \%)$ & 0.1514 \\
\hline Bad rash all over the body & $0(0 \%)$ & I (0.8\%) & $0(0 \%)$ & 0.2625 \\
\hline Severe body allergy & I (0.4\%) & $0(0 \%)$ & $0(0 \%)$ & 0.6307 \\
\hline Burning sensation in the eye & $2(0.8 \%)$ & I (0.8\%) & $0(0 \%)$ & 0.6743 \\
\hline Bone and Muscle Symptoms & $9(3.8 \%)$ & $22(17.7 \%)$ & 7 (7.4\%) & $<0.000 I^{* * *}$ \\
\hline Whole body pain & $2(0.8 \%)$ & $8(6.5 \%)$ & $5(5.3 \%)$ & $0.0084 * *$ \\
\hline Muscle pain & $2(0.8 \%)$ & I (0.8\%) & I (I.I\%) & 0.9764 \\
\hline Muscle relaxation & $0(0 \%)$ & I (0.8\%) & $0(0 \%)$ & 0.2625 \\
\hline Bone pain & $3(1.3 \%)$ & $2(1.6 \%)$ & $0(0 \%)$ & 0.4949 \\
\hline Joint pain & $0(0.0 \%)$ & $5(4.0 \%)$ & I (I.I\%) & $0.0060 * *$ \\
\hline Back pain & $2(0.8 \%)$ & $3(2.4 \%)$ & $0(0 \%)$ & 0.2044 \\
\hline Total Flu-Like Symptoms & 77 (32.5\%) & II 2 (90.3\%) & $29(30.9 \%)$ & $<0.000 I^{* * *}$ \\
\hline Flu symptoms & $26(11.0 \%)$ & $36(29.0 \%)$ & 7 (7.4\%) & $<0.000 I^{* * *}$ \\
\hline Headache & $43(18.1 \%)$ & $48(38.7 \%)$ & $17(18.1 \%)$ & $<0.000 I^{* * *}$ \\
\hline Fever & $3(1.3 \%)$ & $23(18.5 \%)$ & $4(4.3 \%)$ & $<0.000 I^{* * *}$ \\
\hline Chills & $2(0.8 \%)$ & $5(4.0 \%)$ & I (I.1\%) & 0.0772 \\
\hline Sore throat & $2(0.8 \%)$ & $0(0 \%)$ & $0(0 \%)$ & 0.3907 \\
\hline Chest infection & $\mathrm{I}(0.4 \%)$ & $0(0 \%)$ & $0(0 \%)$ & 0.6307 \\
\hline Fatigue Symptoms & $20(8.4 \%)$ & $25(20.1 \%)$ & $3(3.2 \%)$ & $<0.0001 * * *$ \\
\hline Tiredness & $15(6.3 \%)$ & $19(15.3 \%)$ & $3(3.2 \%)$ & $0.217 \mid$ \\
\hline Dizziness and giddiness & $4(1.7 \%)$ & $2(1.6 \%)$ & $0(0 \%)$ & 0.4523 \\
\hline Desire to sleep & I (0.4\%) & $4(3.2 \%)$ & $0(0 \%)$ & $0.0273 *$ \\
\hline GIT Symptoms & $14(5.9 \%)$ & $2(1.6 \%)$ & I (I.1\%) & $0.0383 *$ \\
\hline Nausea and vomiting & $4(1.7 \%)$ & I (0.8\%) & I (I.I\%) & 0.7615 \\
\hline Gastroesophagitis & $2(0.8 \%)$ & $0(0 \%)$ & $0(0 \%)$ & 0.3907 \\
\hline Diarrhea & 7 (3.0\%) & I (0.8\%) & $0(0 \%)$ & 0.1169 \\
\hline Loss of appetite & I (0.4\%) & $0(0 \%)$ & $0(0 \%)$ & 0.6307 \\
\hline Nerve Inflammation Symptoms & $4(1.7 \%)$ & $0(0 \%)$ & $0(0 \%)$ & 0.1563 \\
\hline Bell's palsy & $3(1.3 \%)$ & $0(0 \%)$ & $0(0 \%)$ & 0.2493 \\
\hline Tingling & I (0.4\%) & $0(0 \%)$ & $0(0 \%)$ & 0.6307 \\
\hline Miscellaneous Symptoms & I (0.4\%) & $4(3.2 \%)$ & $0(0 \%)$ & $0.0273 *$ \\
\hline Lymph nodes tenderness and swelling & $0(0 \%)$ & $2(1.6 \%)$ & $0(0 \%)$ & 0.0685 \\
\hline Elevated blood pressure & $0(0 \%)$ & I (0.8\%) & $0(0 \%)$ & 0.2625 \\
\hline Blurred vision & $0(0 \%)$ & $\mathrm{I}(0.8 \%)$ & $0(0 \%)$ & 0.2625 \\
\hline Burning sensation when urinating & I $(0.4 \%)$ & $0(0 \%)$ & $0(0 \%)$ & 0.6307 \\
\hline
\end{tabular}

Notes: Results were offered as frequency (number $(n)$ and percent $(\%))$. Correlation between variables was evaluated using the Chi-square test. $*$ Significant difference at $p \leq$ 0.05 , **significant difference at $\mathrm{p} \leq 0.0 \mathrm{I}$, and $* * *$ significant difference at $\mathrm{p} \leq 0.00 \mathrm{I}$. 
DOSE 1 DOSE 2 DOSE $1 \& 2$

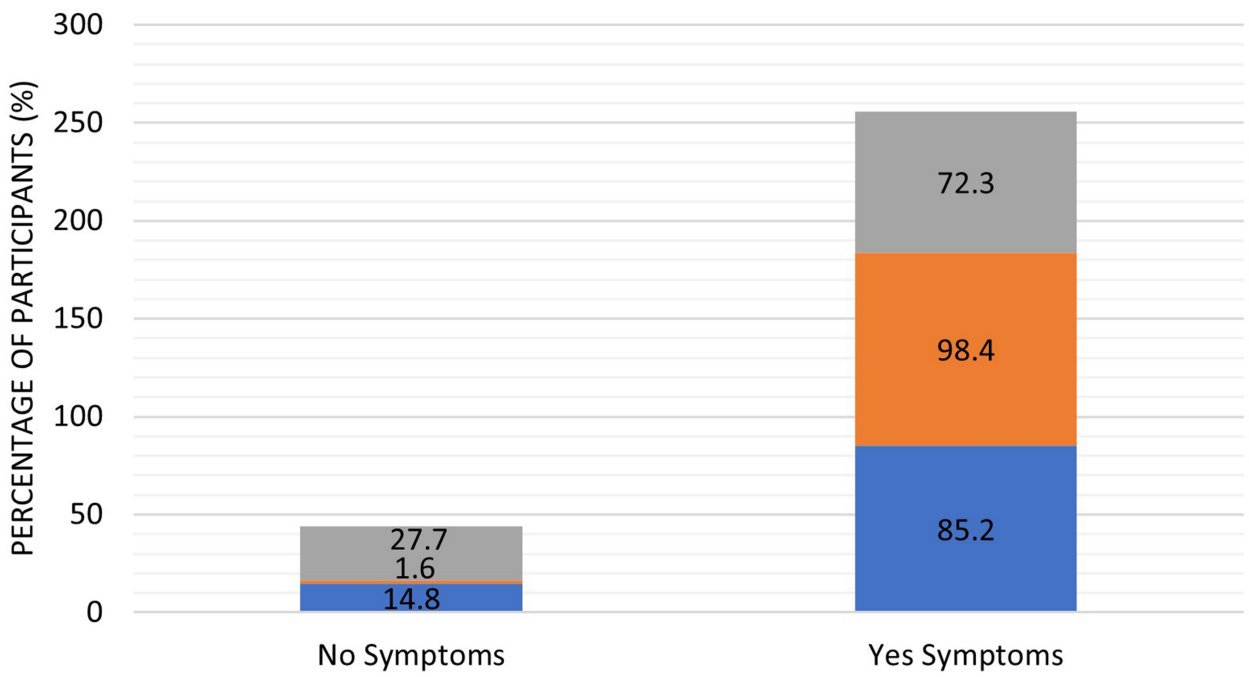

Figure 2 The percentage absence (no symptoms) and presence (yes symptoms) of COVID-19 side effects reported by the study participants following the vaccine's first, second, and both doses. Results were offered as frequency (percentage (\%)).

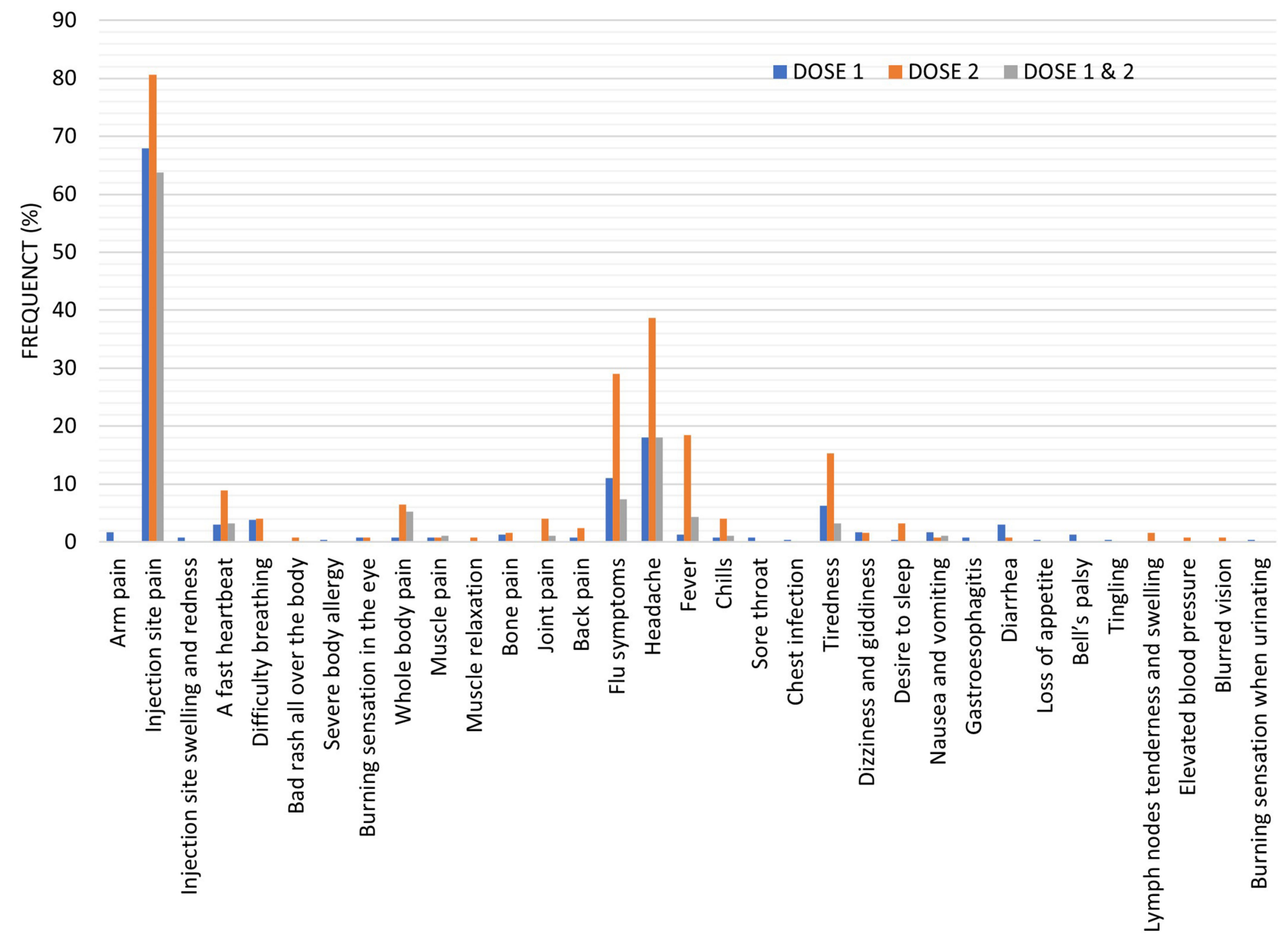

Figure 3 The reported COVID-19 vaccine side effects after the vaccine's first, second, and both doses. Results were offered as frequency (percent (\%)). 
$(68.6 \%)$. Besides, there was a significant increase in the number of people who reported different flu-like symptoms and flu symptoms under 60 years of age, $168(56.2 \%)$ and $59(19.7 \%)$ compared to those over 60 years old, 49 $(31.4 \%)$ and $10(6.4 \%)$. There were no significant differences between the other side effects of the vaccine when comparing their incidence between participants according to their ages.

\section{Correlation Between Presence and Absence of COVID-19 Vaccine Side Effects and the Participant's Sex}

The study results showed a significant difference between the number of males and females who suffered from COVID-19 vaccine side effects. There was a significant increase $(\mathrm{p}<0.001)$ in the number of female participants who reported different side effects after they receive the vaccine (264, 58\%) compared to males $(128,28.1 \%)$. Furthermore, the number of females who reported no side effects $(28,6.2 \%)$ was lower than males $(35,7.7 \%)$, Figure 5.

\section{Reported COVID-19 Vaccine Side Effects Distribution in Participants Previously Infected with Coronavirus versus Non-Previously Infected Participants}

Among the study population, $89 \%$ of participants who had previously been infected with the coronavirus were suffering from some side effects of the COVID-19 vaccine, compared to $86 \%$ of those who had not previously been infected. Of the previously infected participants, 73.7\% complained of local side effects, $5.3 \%$ a fast heartbeat, $26.3 \%$ difficulty breathing, $5.3 \%$ bone pain, $21.1 \%$ flu symptoms, $21.1 \%$ headaches, $10.5 \%$ fever, $5.3 \%$ diarrhea, and $5.3 \%$ Bell's palsy. The distribution of the same side effects among participants who had not previously been infected with the coronavirus was as follows:71.9\% complained of local side effects, $4.6 \%$ a fast heartbeat, $3.9 \%$ difficulty breathing, $1.1 \%$ bone pain, $15.2 \%$ flu symptoms, $23.7 \%$ headaches, $6.5 \%$ fever, $1.8 \%$ diarrhea, and $0.7 \%$ Bell's palsy. The results also showed a significant increase $(\mathrm{p}<0.001)$ in the number of participants who had previously been infected with the coronavirus and suffered from difficulty breathing after receiving the vaccine, compared to participants who had not previously been infected, Figure 6.

\section{Discussion}

Ever since the start of vaccine production, people have expressed worries about their administration's hazards and risks. There is a great variation in people's confidence in vaccines that relies on several factors, including awareness about vaccines, the possible associated risks, experiences, religious, or political aspects, in addition to social and economic status. ${ }^{13}$ Moreover, it has been revealed that people assess vaccination-associated risks compared to other risks in a different way than experts. ${ }^{8-10}$ Some side effects are improbably manifested in pre-licensure clinical studies owing to their minimal frequency, the restricted numbers of participating individuals, and other study restrictions. Therefore, post-marketing monitoring of the side effects following vaccine administration is crucial. ${ }^{14}$

In this study, the data collected from the participating individuals revealed that the adverse effects of the vaccine were reported after two doses, with the majority occurring after the second dose. The most common symptoms are injection site pain, headaches, flu symptoms, fever, and tiredness. Less common side effects are a fast heartbeat, whole body aches, difficulty breathing, joint pain, chills, and drowsiness. Rare side effects included Bell's palsy and lymph nodes tenderness and swelling. Influenza-like symptoms were more common among those under 60 years of age, while pain at the injection site was more common among those who are 60 years and older. These results were in accordance with the FDA Fact Sheet for Recipients and Caregivers. According to the fact sheet, the most common adverse reactions, which include pain at the site of administration, tiredness, headaches, muscle and joint ache, chills, and elevated body temperature, could last for days and were more experienced following the second shot compared to the first one. ${ }^{15}$ This observation could be interpreted on the basis of the immune system's response. The immune system could produce cytokines that could have an inflammatory effect on the blood vessels, muscles, and other tissues. It may also produce flu-like symptoms that last for days after vaccination. This also could explain the prevalence of the side effects development in people of ages below 60 years more than older people as younger people have stronger and more efficient immune systems. The possibility that the Pfizer-BioNTech COVID-19 vaccine could induce a severe allergic reaction is reported. An intense allergic response would eventually take place within a few minutes to one hour following receiving the vaccine dose. Signs of intense allergic response may include 
Table 3 The Reported COVID-19 Vaccine Side Effects and Their Correlation with the Participants Ages

\begin{tabular}{|c|c|c|c|}
\hline & \multicolumn{3}{|c|}{ Frequency (n and \%) } \\
\hline & Age $<60$ (Year) & Age $\geq 60$ (Year) & Chi-Square \\
\hline & $(n=299)$ & $(n=156)$ & p value \\
\hline Absence of Symptoms & $39(13 \%)$ & $24(15.4 \%)$ & 0.5675 \\
\hline Local Symptoms & 209 (69.9\%) & $128(82 \%)$ & $0.0049 * *$ \\
\hline Arm pain & $3(1.0 \%)$ & I (0.6\%) & 1.0000 \\
\hline Injection site pain & $205(68.6)$ & $126(80.8 \%)$ & $0.0056 * *$ \\
\hline Injection site swelling and redness & I (0.3\%) & I (0.6\%) & 1.0000 \\
\hline Hypersensitivity Symptoms & $31(10.4 \%)$ & $9(5.8 \%)$ & 0.1174 \\
\hline A fast heartbeat & $17(5.7 \%)$ & $4(2.7 \%)$ & 0.1614 \\
\hline Difficulty breathing & $10(3.3 \%)$ & $4(2.7 \%)$ & 0.7798 \\
\hline Bad rash all over the body & I (0.3\%) & $0(0 \%)$ & 1.0000 \\
\hline Severe body allergy & $\mathrm{I}(0.3 \%)$ & $0(0 \%)$ & 1.0000 \\
\hline Burning sensation in the eye & $2(0.7 \%)$ & I (0.6\%) & 1.0000 \\
\hline Bone and Muscle Symptoms & $27(9.0 \%)$ & $10(6.4 \%)$ & $0.37 \mid 4$ \\
\hline Whole body pain & $10(3.3 \%)$ & $5(3.2 \%)$ & 1.0000 \\
\hline Muscle pain & $3(1.0 \%)$ & $2(1.3 \%)$ & 1.0000 \\
\hline Muscle relaxation & I (0.3\%) & $0(0 \%)$ & 1.0000 \\
\hline Bone pain & $5(1.7 \%)$ & $0(0 \%)$ & 0.1703 \\
\hline Joint pain & $4(1.3 \%)$ & $2(1.3 \%)$ & 1.0000 \\
\hline Back pain & $4(1.3 \%)$ & I (0.6\%) & 0.6646 \\
\hline Flu Like Symptoms & $168(56.2 \%)$ & 49 (3I.4\%) & $<0.000 I^{* * *}$ \\
\hline Flu symptoms & $59(19.7 \%)$ & 10 (6.4\%) & $<0.000 I^{* * *}$ \\
\hline Headache & 79 (26.4\%) & $29(18.6 \%)$ & 0.0644 \\
\hline Fever & $23(7.7 \%)$ & $7(4.5 \%)$ & 0.2346 \\
\hline Chills & $7(2.3 \%)$ & I $(0.6 \%)$ & 0.2736 \\
\hline Sore throat & $0(0 \%)$ & $2(1.3 \%)$ & 0.1171 \\
\hline Chest infection & $0(0 \%)$ & I $(0.6)$ & 0.3443 \\
\hline Fatigue Symptoms & 35 (11.7\%) & II (7.I\%) & 0.1409 \\
\hline Tiredness & $25(8.4 \%)$ & $10(6.4 \%)$ & 0.5789 \\
\hline Dizziness and giddiness & $6(2.0 \%)$ & $0(0 \%)$ & 0.0988 \\
\hline Desire to sleep & $4(1.3 \%)$ & I (0.6\%) & 0.6646 \\
\hline GIT Symptoms & $12(4.0 \%)$ & $4(2.6 \%)$ & 0.5937 \\
\hline Nausea and vomiting & $4(1.3 \%)$ & I $(0.6 \%)$ & 0.6646 \\
\hline Gastroesophagitis & $0(0 \%)$ & $2(1.3 \%)$ & 0.1171 \\
\hline Diarrhea & $7(2.3 \%)$ & I $(0.6 \%)$ & 0.2736 \\
\hline Loss of appetite & I (0.3\%) & $0(0 \%)$ & 1.0000 \\
\hline Nerve Inflammation Symptoms & $3(1.0 \%)$ & I $(0.6 \%)$ & 1.0000 \\
\hline Bell's palsy & $3(1.0 \%)$ & $0(0 \%)$ & 0.5544 \\
\hline Tingling & $0(0 \%)$ & $\mathrm{I}(0.6 \%)$ & 0.3443 \\
\hline Miscellaneous Symptoms & $4(1.3 \%)$ & I (0.6\%) & 0.6646 \\
\hline Lymph nodes tenderness and swelling & $2(0.7 \%)$ & $0(0.6 \%)$ & 0.5484 \\
\hline Elevated blood pressure & I (0.3\%) & $0(0 \%)$ & 1.0000 \\
\hline Blurred vision & I $(0.3 \%)$ & $0(0 \%)$ & 1.0000 \\
\hline Burning sensation when urinating & $0(0 \%)$ & I (0.6\%) & 0.3443 \\
\hline
\end{tabular}

Notes: Results were offered as frequency (number $(\mathrm{n})$ and percent $(\%))$. Correlation between variables was evaluated using the Chi-square test. **Significant difference at $p \leq 0.01$, and $* * *$ significant difference at $p \leq 0.001$. 


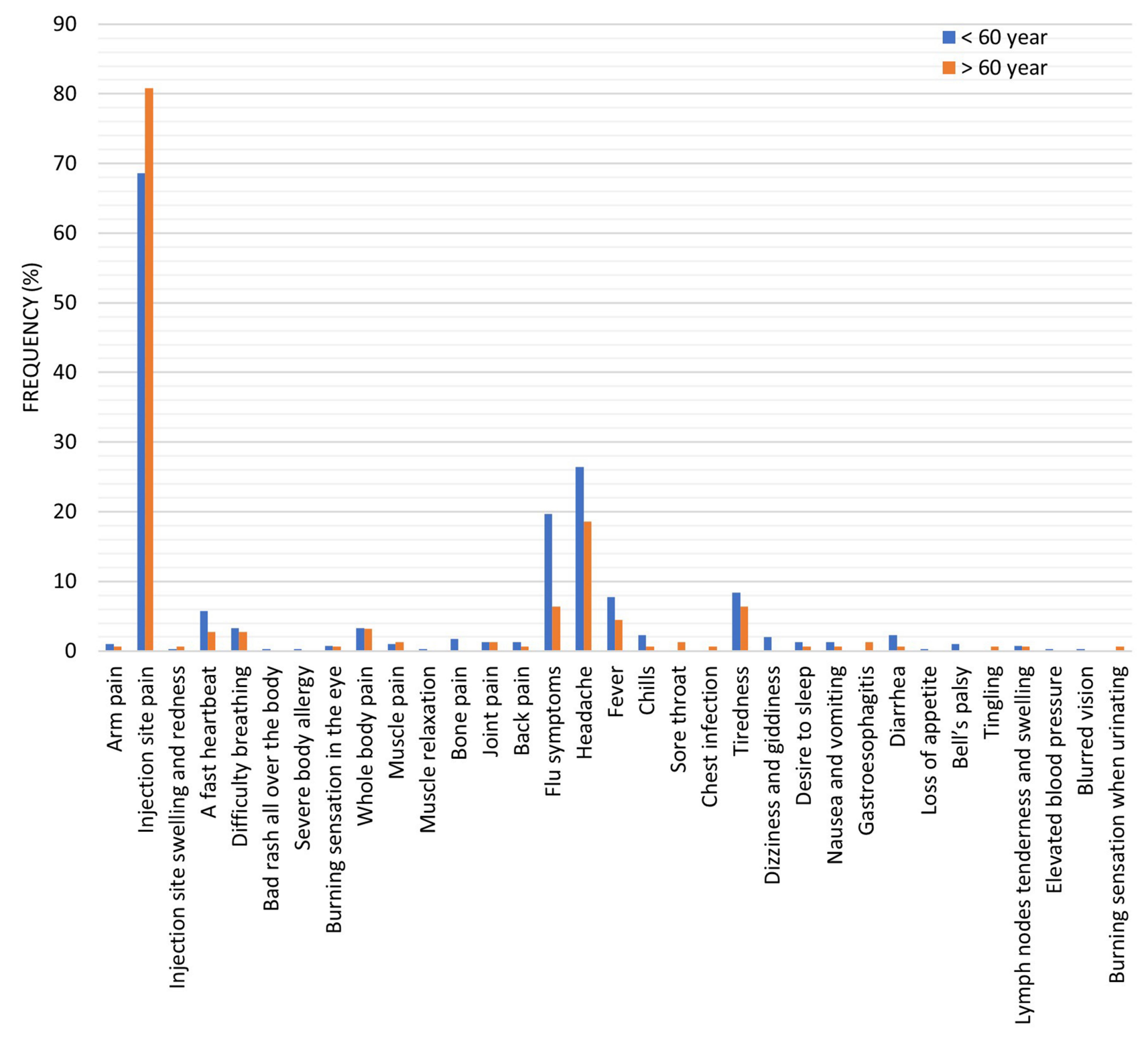

Figure 4 The reported COVID-19 vaccine side effects distribution according to the participants' ages. Results were offered as frequency (percent (\%)).

dyspnea, face and throat swelling, rapid heartbeat, body rash, dizziness and weakness. Bell's palsy has also been reported as a rare side effect for the vaccine. ${ }^{16,17}$ The results of the present study showed a significant increase in the number of individuals who reported hypersensitivity symptoms after receiving the second dose of the vaccine. A minor percentage of individuals have also developed Bell's palsy. These results were in agreement with the previously reported side effects for the vaccine.

This study results showed that injection site pain was recorded more often among individuals 60 years and older (80.8\%) than among younger individuals (68.6\%). A remarkably reduced percentage of individuals proclaimed injection-site swelling and redness. The percentage of individuals suffering local symptoms significantly increased after the second dose. Our results disagree with the results recorded by Polack et al ${ }^{18}$ who reported that injection site pain was more frequent among people younger than 55 years compared to those who are older. They also noticed that the percentages of people reporting local symptoms after the first and the second doses of the vaccine were nearly the same. However, our study and their study reported the same results concerning injection-site swelling and redness. Our results agree with Polack et $\mathrm{al}^{18}$ as the two studies reported that the vaccine-associated systemic side effects 


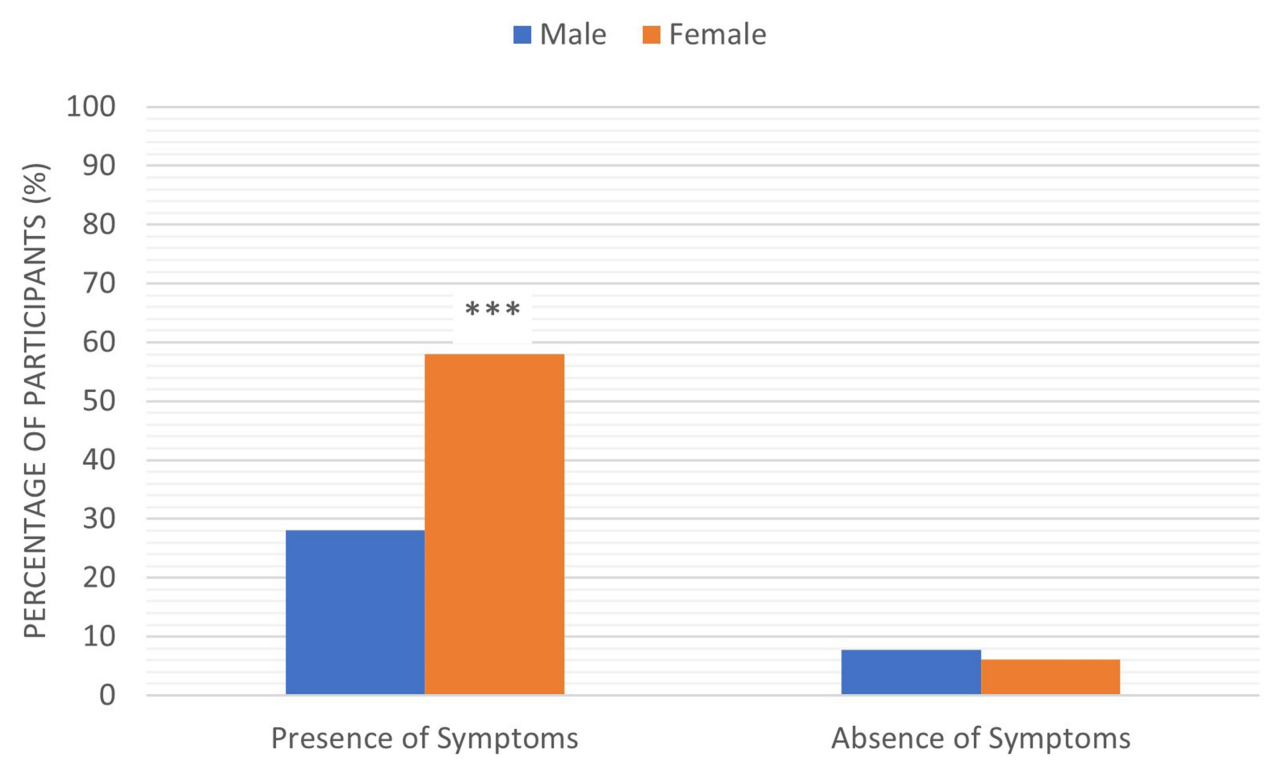

Figure 5 The correlation between presence and absence of COVID-19 vaccine side effects and the participant's sex. Results were offered as frequency (percent (\%)). Correlation between variables was evaluated using the Chi-square test. $* * *$ Significant difference at $p \leq 0.00$ I.

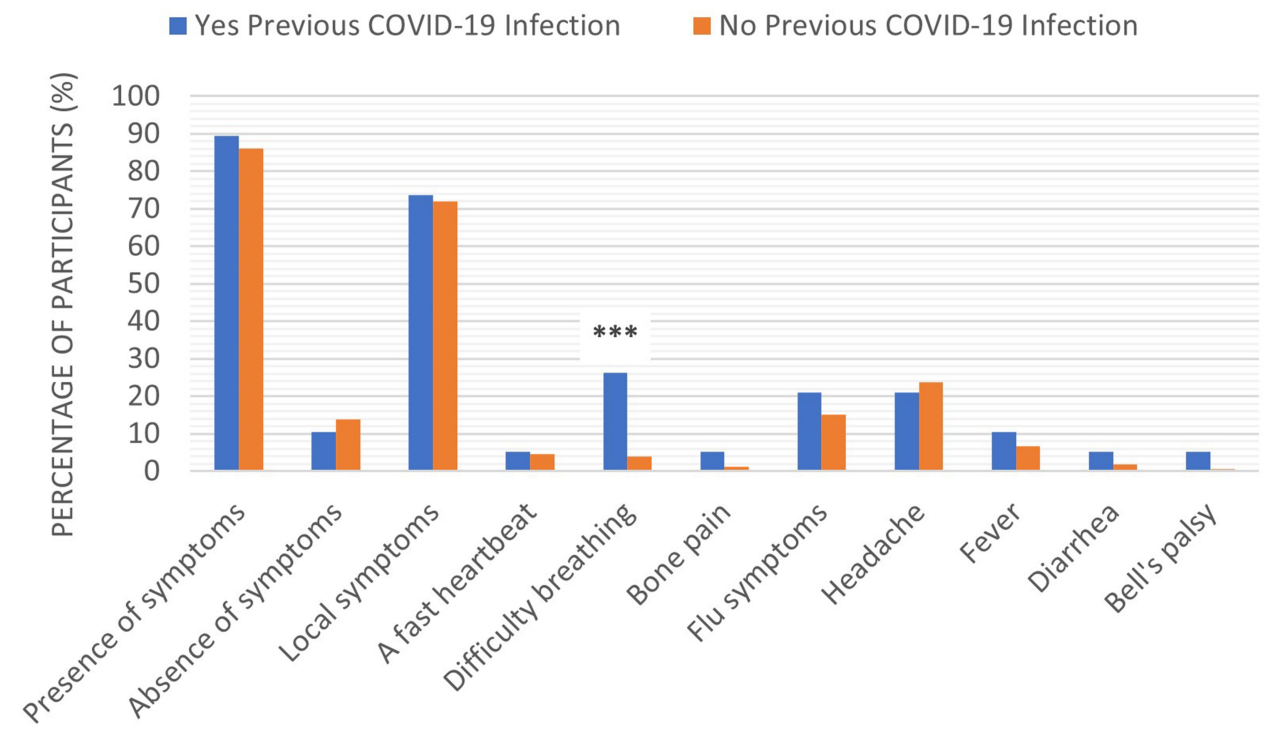

Figure 6 The reported COVID-19 vaccine side effects distribution in participants previously infected with coronavirus versus non-previously infected participants. Results were offered as frequency (percent $(\%)$ ). Correlation between variables was evaluated using the Chi-square test. *** Significant difference at $\mathrm{p} \leq 0.00 \mathrm{I}$.

were more common among younger people and more common after the second vaccine dose. The two studies reported that headaches were the most common symptom after a vaccine injection. Also, fever was most common among young people after the second dose.

\section{Limitations and Strengths}

The current study is one of the leading studies that shed light on the side effects of the Pfizer-BioNTech COVID-19 vaccine. Few or no studies have documented the vaccine's side effects, especially the post-marketing follow-up of the vaccine. The limitation of the current study is that it monitored the vaccine's short-term side effects (immediately after injection). In contrast, the medium and long-term effects of the vaccine are still unknown. Furthermore, the study was conducted only on Arab participants only, thus excluding other races. The small number of the study participants who were previously infected with the coronavirus suggest the need for future studies with a suitable and compatible sample size for both previously infected and non-infected persons. 


\section{Conclusion}

In general, the side effects that were identified from the Pfizer-BioNTech COVID-19 vaccine are the typical symptoms of most previous vaccines, and most of them are tolerated. ${ }^{19,20}$ Also, most of the symptoms have been reported by the manufacturing company and the FDA Facts Sheet. ${ }^{15}$ However, for some of people it is mandatory to monitor them for a short time immediately after receiving their vaccine doses.

\section{Data Sharing Statement}

All relevant data are available in the present manuscript. The data presented in this study are available on request from the corresponding author.

\section{Ethics Approval and Consent to Participate}

The study protocol was approved by the Unit of Biomedical Ethics Research Committee, Faculty of Medicine, King Abdulaziz University, Saudi Arabia (Reference No. 111/21). The study was also approved from the Public Health Research and Health Statistics Saudi Center for Disease Control and Prevention (SCDC), Saudi Arabia (Registration number: (202,102,221; IRB number: 111-21). Concerning the informed consent statement, the study participants were informed that as they completed the questionnaire, they were accepted to be enrolled in the study. This study was conducted in accordance with the Declaration of Helsinki.

\section{Acknowledgments}

All the authors are deeply thankful to everyone who complete the questionnaire.

\section{Disclosure}

The authors declare no conflicts of interest.

\section{References}

1. World Health Organization. Coronavirus disease (COVID-19) situation report-127. World Health Organisation (WHO); 2020:1-17. Available from: https://apps.who.int/iris/handle/10665/332232. Accessed February 20, 2021.

2. Cucinotta D, Vanelli M. WHO declares COVID-19 a pandemic. Acta Biomed. 2020;91:157-160. doi:10.23750/abm.v91i1.9397

3. World Health Organisation. Draft landscape and tracker of COVID-19 candidate vaccines. World Health Organisation (WHO); 2020. Available from: https://www.who.int/publications/m/item/draftlandscape-of-covid-19-candidate-vaccines. Accessed February 20, 2021.
4. FDA Briefing Document. Pfizer-BioNTech COVID-19 vaccine. U.S. Food and Drug Administration; 2020:1-53. Available from: https:// www.fda.gov/media/144245/download. Accessed February 11, 2021.

5. FDA Briefing Document. Moderna COVID-19 Vaccine. U.S. Food and Drug Administration; 2020:1-54. Available from: https://www. fda.gov/media/144434/download. Accessed February 11, 2021.

6. Centers for Disease Control and Prevention. Understanding how COVID-19 vaccines work. Vaccines. 2021. Available from: https:// www.cdc.gov/coronavirus/2019-ncov/vaccines/different-vaccines /how-they-work.html. Accessed February 11, 2021.

7. Centers for Disease Control and Prevention. What to expect at your appointment to get vaccinated for COVID-19| CDC. Vaccines. 2021. Available from: https://www.cdc.gov/coronavirus/2019-ncov/vac cines/expect.html. Accessed February 11, 2021.

8. Davis TC, Fredrickson DD, Arnold CL, et al. Childhood vaccine risk/ benefit communication in private practice office settings: a national survey. Pediatrics. 2001;107:e17-e17. doi:10.1542/peds.107.2.e17

9. Gust DA, Woodruff R, Kennedy A, Brown C, Sheedy K, Hibbs B. Parental perceptions surrounding risks and benefits of immunization. Semin Pediatr Infect Dis. 2003;14:207-212. doi:10.1016/S10451870(03)00035-9

10. Bond L, Nolan T, Pattison P, Carlin J. Vaccine preventable diseases and immunisations: a qualitative study of mothers' perceptions of severity, susceptibility, benefits and barriers. Aust $N$ Z J Public Health. 1998;22:441-446. doi:10.1111/j.1467-842X.1998.tb01411.x

11. MacDonald NE, Eskola J, Liang X, et al. Vaccine hesitancy: definition, scope and determinants. Vaccine. 2015;33:4161-4164. doi:10.1016/j.vaccine.2015.04.036

12. Aranda S. Ten Threats to Global Health in 2019. World Health Organisation (WHO); 2019:1-18.

13. Larson H, Cooper L, Eskola J, Katz S, Ratzan S. Addressing the vaccine confidence gap. Lancet. 2011;378(9790):526-535. doi:10.1016/S0140-6736(11)60678-8

14. Wessely S. Emergency preparedness and response. J R Army Med Corps. 2018;166:jramc-2018-000965. doi:10.1136/jramc-2018-000929

15. Pfizer COVID-19 vaccine EUA fact sheet for healthcare providers administering vaccine (vaccination providers). 2021:1-30. Available from: https://www.fda.gov/media/144413/download. Accessed March 31, 2021.

16. Crist C. Track vaccine recipients for facial paralysis. Available from: https://www.webmd.com/vaccines/covid-19-vaccine/news/20201217/ fda-says-vaccine-recipients-should-be-monitored-for-facial-paralysis. Accessed February 20, 2021.

17. What we know about a link between bell's palsy and COVID-19 vaccines. Available from: https://www.businessinsider.com/what-weknow-link-between-bells-palsy-covid-19-vaccines-2020-12. Accessed February 20, 2021.

18. Polack F, Thomas S, Kitchin N, et al. Safety and efficacy of the BNT162b2 mRNA covid-19 vaccine. $N$ Engl $J$ Med. 2020;383:2603-2615. doi:10.1056/NEJMoa2034577

19. Van Balveren-slingerland L, Rümke H, Kant A. Reported adverse events following influenza vaccination. Ned Tijdschr Geneeskd. 2014;158:A6841.

20. Zhou W, Pool V, Iskander J, et al. Surveillance for safety after immunization: Vaccine Adverse Event Reporting System (VAERS)United States, 1991-2001. MMWR CDC Surveill Summ. 2003;52:1-24 


\section{Publish your work in this journal}

The International Journal of General Medicine is an international, peer-reviewed open-access journal that focuses on general and internal medicine, pathogenesis, epidemiology, diagnosis, monitoring and treatment protocols. The journal is characterized by the rapid reporting of reviews, original research and clinical studies

across all disease areas. The manuscript management system is completely online and includes a very quick and fair peer-review system, which is all easy to use. Visit http://www.dovepress.com/ testimonials.php to read real quotes from published authors.

Submit your manuscript here: https://www.dovepress.com/international-journal-of-general-medicine-journal 